\title{
Experience in multicatheter interstitial high-dose-rate breast brachytherapy: dose-volume histogram analysis of the first results
}

\author{
Alena Demianovich!, Dmitriy Sanin, PhD!, Valeriya Martynova!, Natalia Borysheva, PhD!', Aleksandr Obuhov, MD, PhD!', \\ Gamzat Aminov, MD, PhDl, Prof. Igor Gulidov, MD, PhD!, Yuriy Mardyntsky, MD, PhD, Cor. Mem. of RAS!, \\ Marina Kiseleva, MD, PhD', Sergey Ivanov, MD, PhD, Cor. Mem. of RAS', Prof. Andrey Kaprin, MD, PhD² \\ 'A. Tsyb Medical Radiological Research Centre - branch of the National Medical Research Radiological Centre of the Ministry of Health of \\ the Russian Federation, Obninsk, Russia, ${ }^{2}$ National Medical Research Radiological Centre of the Ministry of Health of the Russian Federation, \\ Obninsk, Russia
}

\begin{abstract}
Purpose: To report characteristics of our treatment scheme of high-dose-rate (HDR) brachytherapy of breast cancer and to show the first outcomes of dosimetric planning analysis based on dose-volume histogram (DVH).

Material and methods: Since August 2017, 25 patients diagnosed with T1N0M0 breast cancer have received a treatment in our center. There was lumpectomy and following breast HDR brachytherapy (10 fractions of 3.4 Gy) administered to each patient. A planning target volume (PTV) and organs at risk (OARs) were recorded with DVH analysis.

Results: The study describes the full procedure of breast HDR brachytherapy with the lumpectomy. Twenty-five patients were treated, including 9 with cancer of the left breast and 16 of the right breast. The median age was 65 years. The first analysis of DVH data shows that the main OARs were ribs and skin. Mean value of $\mathrm{D}_{\max }$ (ribs) for all patients was 19.90 Gy (55.88\% of prescribed dose) and for the skin $30.88 \mathrm{~Gy}$ ( $90.74 \%$ of prescribed dose). During the treatment, there was only one case of toxic effects, which was pigmentation on the skin due to excess of dose limit of 1.4 Gy. Therefore, the limit exceeding of $1 \mathrm{~Gy}$ does not give any significant toxic effects.

Conclusions: This study is the first stage of the dosimetric evaluation of a new method. The analysis allows treating complex localizations of the breast cancer, for example, in a close position to the skin or ribs. In order to minimize the toxic effects, it is necessary to consider patient selection, catheter administration, and dose optimization.

J Contemp Brachytherapy 2019; II, 4: 349-355 DOI: https://doi.org/10.5114/jcb.2019.87024
\end{abstract}

Key words: breast cancer, lumpectomy, high-dose-rate brachytherapy, radiation therapy, dosimetric parameters.

\section{Purpose}

Breast cancer is the most common malignancy among women all over the world. In 2015, there were about 2.4 million cases among the total population, including about 44,000 of male cases $[1,2]$.

Today, not all causes of breast cancer are known, but lifestyle and reproductive factors play a significant roles. Low screening rates and incomplete reporting can decrease breast cancer rates in developing countries, which can explain some of the differences. Breast cancer is the leading cause of cancer mortality among women around the world. The World Health Organization (WHO) declares that more than 2 million new cases and up to 600,000 deaths from breast cancer could be detected among people worldwide in 2018 [3,4].
Currently, there are many different types of treatment for patients with breast cancer, including local (surgery, radiation therapy) and systemic (chemotherapy, hormonotherapy, and immunotherapy).

Therapeutic tactics should be individual, taking into account a number of prognostic factors. That is why it is so important to specify a direction of treatment, which can be radical, palliative, or symptomatic. In the preoperative period, it is necessary to assess the resorption degree of tumor and to determine the scope of surgery. After the operation and according to histological results, it is necessary to establish a post-operative treatment plan, with chemo-, hormonal, and/or radiation therapy.

Surgery, as the only method of treatment of patients with regional metastases, leads to frequent occurrence
Address for correspondence: Natalia Borysheva, PhD, A. Tsyb Medical Radiological Research Centre - branch of the National Medical Research Radiological Centre of the Ministry of Health of the Russian Federation, Obninsk, Russia, e-mail: borysheva@yandex.ru
Received: 12.02 .2019

Accepted: 09.07.2019

Published: 29.08.2019 
and reoccurrence of distant metastases. In order to improve the immediate and long-term results of surgical treatment, in 1901, the idea of using radiation therapy was developed [4].

The main types of post-operative irradiation are: - external beam radiotherapy (EBRT), with total dose of 45-50 Gy and single fraction of 1.8-2 Gy;

- boost - additional dose to tumor bed complementary to EBRT of the whole breast (total dose ranges from 8 to $20 \mathrm{~Gy}$ );

- brachytherapy, which can be carried out using high-, low-, or pulsed-dose-rate sources, delivered with single or multicatheter technique $[5,6]$.

Recent observations show a tendency towards significant rejuvenation of malignant tumors in this localization. That is why organ preservation treatments become more relevant and are essential to be developed [7]. Also, there is a tendency in minimizing treatment time, injury time, and toxicity, and this is why brachytherapy is the most promising method of treatment in the early stages. The main purpose of such irradiation is reducing the risk of local recurrence and sparing the surrounding normal tissues and organs at risk (OARs). Breast accelerated partial breast irradiation (APBI) has a number of potential advantages over whole breast EBRT:

- possibility to start therapy in a few days after lumpectomy;

- irradiation with a higher dose in a shorter period during post-operative therapy;

- irradiation delivered to a small volume of breast around tumor bed;

- significantly lower radiation exposure to OARs and normal tissues;

- reduced amount of errors due to a source small step and anatomical changes (e.g., patient's breathing) [8].

The feasibility and efficacy of APBI were extensively evaluated in many scientific publications. Moreover, the advantages of application of this method for patients with early stage breast cancer were also presented, with

Table 1. Patient selection criteria for accelerated partial breast irradiation (APBI) of breast cancer

\begin{tabular}{ll}
1 & Age $\geq 50$ years \\
\hline 2 & Tumor size $\leq 2 \mathrm{~cm}$ \\
\hline 3 & $\begin{array}{l}\text { Invasive non-specific type of cancer, infiltrative non-spe- } \\
\text { cific type of cancer }\end{array}$ \\
\hline 4 & Grade I or II of malignancy \\
\hline 5 & $\begin{array}{l}\text { The absence of lymph node lesions and distant metasta- } \\
\text { ses, NO and MO }\end{array}$ \\
\hline 6 & Surgical margin: cancer not exposed, $\geq 3 \mathrm{~mm}$ \\
\hline 7 & $\begin{array}{l}\text { Luminal A (ER+, PR+, Her } 2 / \text { neu+1) and luminal B } \\
\text { (ER+/, PR+/, Her2/neu+1) }\end{array}$ \\
\hline 8 & Negative results for mutations of BRCA genes \\
\hline 9 & \begin{tabular}{l} 
The Ki-67 $\leq 40 \%$ \\
\hline
\end{tabular}
\end{tabular}

$E R$ - estrogen receptors, $P R$ - progesterone receptors, Her2/neu - human epidermal growth factor receptor 2, Ki-67-antigen identified by monoclonal antibody Ki-67 different types of fractionations. The main ones included $8 \times 4$ Gy, 7 × 4.3 Gy (GEC-ESTRO recommendations), and 10 fractions of 3.4 Gy (ABS recommendations) per fraction twice a day, with a break of at least 6 hours as well as $7 \times 5.2$ Gy and $6 \times 6$ Gy fractions. In recent years, studies on single fraction irradiation of 16-20 Gy were published $[6,8,9,10,11,12]$

In 2017, the protocol for the breast cancer treatment with high-dose-rate (HDR) brachytherapy in the mode of $10 \times 3.4$ Gy for 5 days, 2 fractions per day with a break of at least 6 hours was adopted.

\section{Material and methods}

Between August 2017 and November 2018, twentyfive patients with T1N0M0 stage breast cancer were treated in the A. Tsyb Medical Radiological Research Centre - branch of the National Medical Research Radiological Centre of the Ministry of Health of the Russian Federation.

Patients were selected by a multidisciplinary team, which consisted of the operating surgeon, mammologist, and radiation oncologist. The radiation oncologist examined the patients several days before the surgery, within a few days after, and at the patient's discharge. The spread of the tumor process was assessed by palpation, mammography, and ultrasound (US) examination. In all cases, the diagnosis was confirmed morphologically, the immunohistochemical test was carried out, and the molecular subtype of the tumor was determined. Such anamnesis is necessary to exclude relative and absolute contraindications for HDR APBI, including acute infectious disease, the presence of human immunodeficiency viruses (HIV), Wassermann reaction (RW), hepatitis B surface antigen ( $\mathrm{HbsAg})$, hepatitis $\mathrm{C}$ virus $(\mathrm{HCV})$ in the acute stage, pregnancy or lactation, severe competing (somatic) diseases incompatible with brachytherapy, and mental illnesses that could lead to inability to comply allowed radiation doses for critical structures.

Selection criteria of patients were chosen after careful analysis of the GEC-ESTRO and the American Brachytherapy Society (ABS) recommendations as well as the results of other studies $[6,9,10]$. The final criteria are presented in Table 1. Tumor and patients' characteristics are shown in Table 2.

Before lumpectomy, all patients were sent for US examination, where it was necessary to put an intraoperative mark on the skin for operating surgeon and radiologist. After that, a spiral computed tomography (CT) scan was performed, with oncologist, radiologist, and medical physicist direct attendance. They determined the location and size of the tumor, and discussed the preliminary number of introducers and their configuration. The tumor with the capture of healthy tissue (at least one centimeter) and the sentinel lymph node are removed during the lumpectomy, and for more accurate positioning of the tumor during post-operative examination and planning, the surgeon must install the metal mark into the tumor bed.

A few days later, post-operative CT examination was carried out. At this stage, a medical physicist and oncologist assessed the introducers' location and started to 
Table 2. Patient and tumor characteristics

\begin{tabular}{|c|c|c|}
\hline 1 & Age & Mean: 65 years \\
\hline 2 & Tumor size & Mean: $0.9 \mathrm{~cm}$ \\
\hline 3 & Type of cancer & $\begin{array}{l}\text { Invasive non-specific type of cancer: } 73 \% \\
\text { Infiltrative non-specific type of cancer: } 27 \%\end{array}$ \\
\hline 4 & Degree of malignancy & $\begin{array}{c}\text { I: } 40 \% \\
\text { II: } 60 \% \\
\end{array}$ \\
\hline 5 & The absence of lymph node lesions and distant metastases & $100 \%$ \\
\hline 6 & Surgical margin: cancer not exposed, $\geq 3 \mathrm{~mm}$ & $100 \%$ \\
\hline 7 & Luminal type of cancer & $\begin{array}{l}\text { Luminal A: } 80 \% \\
\text { Luminal B: } 20 \%\end{array}$ \\
\hline 8 & Negative results for mutations of BRCA genes & $100 \%$ \\
\hline 9 & Ki-67 & $\begin{array}{l}\text { Mean: } 11 \% \\
\text { Median: } 8 \%\end{array}$ \\
\hline
\end{tabular}

create a treatment plan. However, the patient was finally allowed for irradiation only after it has been confirmed that there was a clear surgical margin and that there was no histological evidence of axillary metastasis.

It should be noted that the patient position during CT scan before and after surgery as well as throughout the treatment must always be the same.

The fractionation type and constraints of tolerant doses to the OARs were carefully thought over and developed after analyzing an international recommendations and discussing by the multidisciplinary team.

Doses are normalized in accordance with international standards. They are converted into an equivalent dose of 2 Gy per fraction (EQD2) using Formula 1:

$$
E Q D_{2}=n \cdot d \cdot \frac{d+\left(\frac{\alpha}{\beta}\right)}{2+\left(\frac{\alpha}{\beta}\right)}
$$

where $n$ is the number of fractions, $d$ is the dose per fraction (in Gy), and $\alpha / \beta$ is 3 for OARs and 4 for the tumor. There is vast amount of data on different values of the $\alpha / \beta$ for breast, but it is considered that this parameter is low enough to use higher doses in a shorter period of irradiation (hypofractionation).

The value of $\alpha / \beta=4$ was chosen as the most suitable, with the total dose and falling under the GEC-ESTRO recommendations $[6,8,12,13,14,15]$. A model of fractionation agreed with the ABS recommendations: $3.4 \mathrm{~Gy}$ per fraction, 2 times a day for 5 days, with a break of at least 6 hours [10].

Initially, gross tumor volume (GTV) was drawn for modelling of the dose distribution. In our case, it was a nominal volume and position of tumor before surgery. Also, our GTV was nearly equal to the related target volume (ImTV) and estimated tumor bed (ETB) in the GEC ESTRO recommendations [16]. Mammograms, US, and $\mathrm{CT}$ images have provided important information about the tumor size and localization; the tumor affected quadrant, and the distances between tumor and skin and tumor and the chest wall were evaluated. After that, it was possible to consider catheter implantation. At the same time, the surgical bed mark placement helped to determine the tumor bed after lumpectomy on the axial CT images. It is important to note that catheters are installed during an open cavity surgery, and the target volume is better visible with a seroma. Unfortunately, the seroma is not always possible to visualize, as in our study on the post-operative CT examination few days after surgery.

The clinical target volume (CTV) was defined as GTV plus $1 \mathrm{~cm}$ radial expansion, and planning target volume (PTV) was CTV plus $0.5 \mathrm{~cm}$ in order to avoide possible errors in target positioning. Figure 1A shows the location of the tumor used for preoperative planning of introducers' installation. Figure 1B demonstrates the introducers' placement as well as the irradiation target contour. CTV and PTV were limited to the chest wall and were $5 \mathrm{~mm}$ below the skin surface [17]. The ribs, skin, and lungs were the main OARs, and were contoured in all cases. The heart and liver could be individually contoured depending on the tumor bed location.

To control the quality assurance of brachytherapy, dose restrictions were established. They are presented in Table 3 , and include $D_{\text {mean }}$ and $D_{\text {max }}$ to the OARs, where $\mathrm{D}_{\max }$ is the dose at the hot point and it is calculated based on the dose-volume histogram (DVH).

A device for contact radiation therapy GammaMedplus iX HDR/PDR aflerloader with a ${ }^{192}$ Ir source was used (Varian, USA) during the radiation therapy. The physical parameters of the source are presented in Table 4 . The medical physicist used volume optimization to PTV for breast brachytherapy planning and after that, the plan was improved manually. Dosimetric 3D-planning was carried out using TG-43 (BrachyVision planning system, Varian, USA).

\section{Results}

Twenty-five patients were treated with HDR brachytherapy, including 9 patients with cancer of the left breast and 16 of the right breast. Median age was 65 years (range, 55-78). However, one patient was removed from the study due to the significant displacement of the mark. 

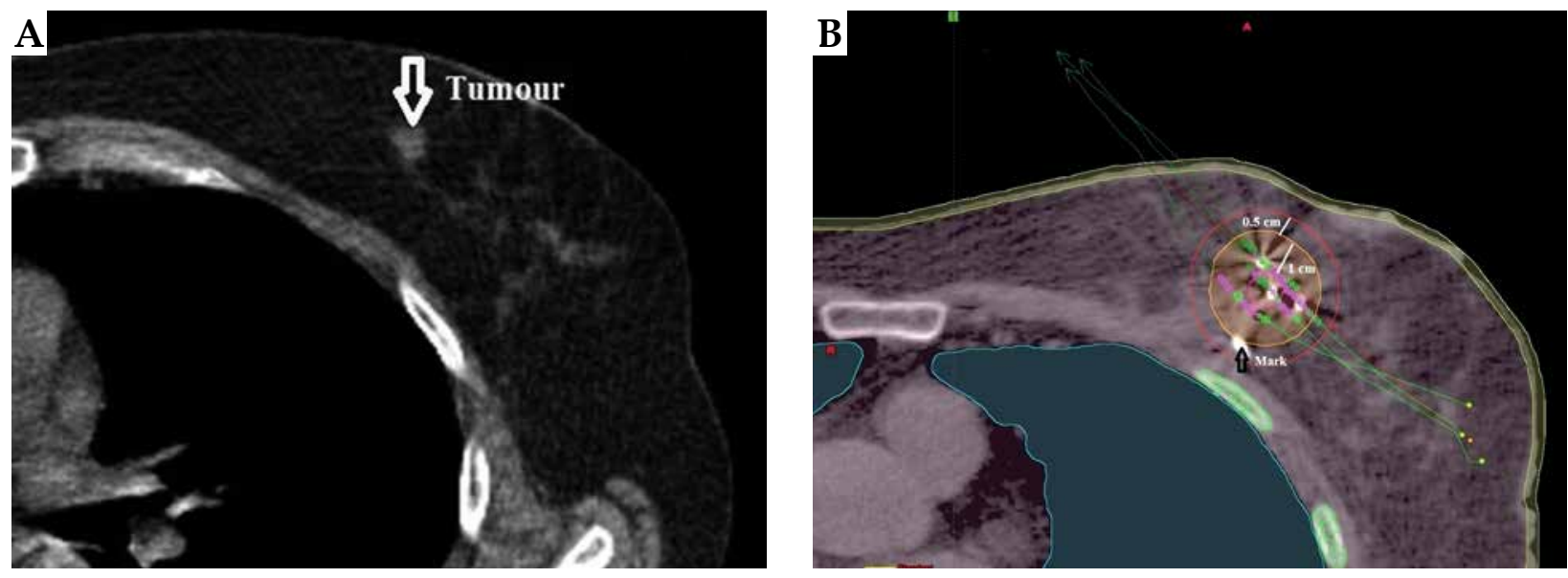

Fig. 1. A) Computed tomography image of tumor position before lumpectomy; B) The contouring example of a patient after introducer administration. Purple line shows GTV, orange line is CTV, and red line is the contour of PTV. The arrow shows the intraoperative mark

Table 3. Recommended dose-volume constraints for target volume and organs at risk (OARs) PTV Constraints $\left[\mathrm{cm}^{3}\right]$

\begin{tabular}{lc}
\hline $\mathrm{V}_{90}$ & $\geq 90 \%$ absolute PTV volume \\
\hline Organ at risk & Constraints (from prescribed dose) [Gy] \\
\hline Skin & $D_{\max } \leq 100 \%$ \\
\hline Ribs & $D_{\max } \leq 145 \%$ \\
\hline Lung & $D_{\max } \leq 60 \%$ \\
\cline { 2 - 2 } & $D_{\operatorname{mean}} \leq 8 \%$ \\
\hline Heart & $D_{\max } \leq 50 \%$ \\
\hline Liver & $D_{\operatorname{mean}} \leq 8 \%$ \\
\hline
\end{tabular}

This fact did not allow to determine the target position accurately.

For 25 patients, the mean value of PTV was $56.28 \mathrm{~cm}^{3}$, the mean value of $V_{90}$ was $91.50 \%$, and the mean value of $\mathrm{V}_{100}$ was $89.50 \%$ for all patients. Occasionally, better values of $\mathrm{V}_{100}$ and $\mathrm{V}_{90}$ were changed in favor to reduce

Table 5. Values of the $D_{\text {mean }}$ to organs at risk (OARs)

\begin{tabular}{lcc} 
Organ at risk & $\begin{array}{c}\text { Mean value of dose } \\
\text { for all patients [Gy] }\end{array}$ & $\begin{array}{c}\text { Percentage of the } \\
\text { prescribed doses [\%] }\end{array}$ \\
\hline Heart & $D_{\max }=3.97$ & 11.15 \\
\cline { 2 - 3 } & $D_{\text {mean }}=1.05$ & 3.08 \\
\hline Liver & $D_{\text {max }}=11.48$ & 33.76 \\
\hline Lung & $D_{\text {max }}=15.50$ & 45.59 \\
\cline { 2 - 3 } & $D_{\text {mean }}=1.27$ & 3.74 \\
\hline Ribs & $D_{\text {max }}=19.90$ & 55.88 \\
\hline Skin & $D_{\text {max }}=30.85$ & 90.74
\end{tabular}

Table 4. Physical parameters for ${ }^{192} \mathrm{Ir}$

\begin{tabular}{lc} 
Parameter & Value \\
\hline Half-life & 2.4 months \\
\hline Initial source strength & $370 \mathrm{GBq}$ \\
\hline Dose rate constant & $108 \mathrm{cGy} / \mathrm{hU}$ \\
\hline Air kerma rate constant & $1.108 \mathrm{mGy} / \mathrm{h}$ \\
\hline$E_{\text {max }, \beta}$ & $0.675 \mathrm{MeV}$ \\
\hline$E_{\text {mean }, \beta}$ & $0.181 \mathrm{MeV}$ \\
\hline$E_{\beta}$ & $0.136-1.062 \mathrm{MeV}$ \\
\hline$E_{\text {mean }, \gamma}$ & $0.375 \mathrm{MeV}$
\end{tabular}

the dose to normal tissues and OARs (Table 5). Skin and ribs were the main OARs. Based on the criterion adopted in the protocol, $\mathrm{D}_{\max }$ attributable to the skin were not higher than $100 \%$ of the prescribed dose (34 Gy for the whole course). Figure 2 presents DVH results of the skin for all of the 25 patients. $D_{\max }$ for patients' number 2, 10, and 24 were higher than allowable dose, and the total skin doses for these patients were $35 \mathrm{~Gy}\left(\mathrm{EQD}_{2}, 45.5 \mathrm{~Gy}\right)$, $35.2\left(\mathrm{EQD}_{2}, 45.9\right)$, and $35.4\left(\mathrm{EQD}_{2}, 46.3\right)$, respectively. The patient number 24 had a pigmentation, and the maximum excess of the skin dose over the allowable dose was

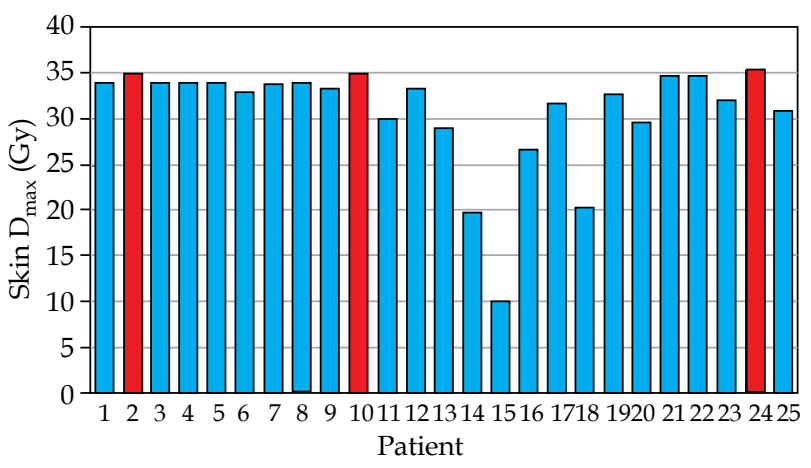

Fig. 2. $D_{\max }$ values to the skin for all of the 25 patients 


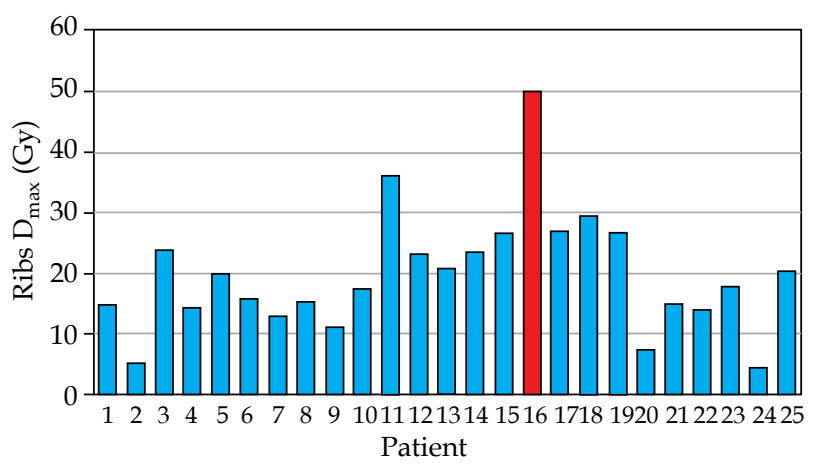

Fig. 3. $\mathrm{D}_{\max }$ values to the ribs for all of the 25 patients

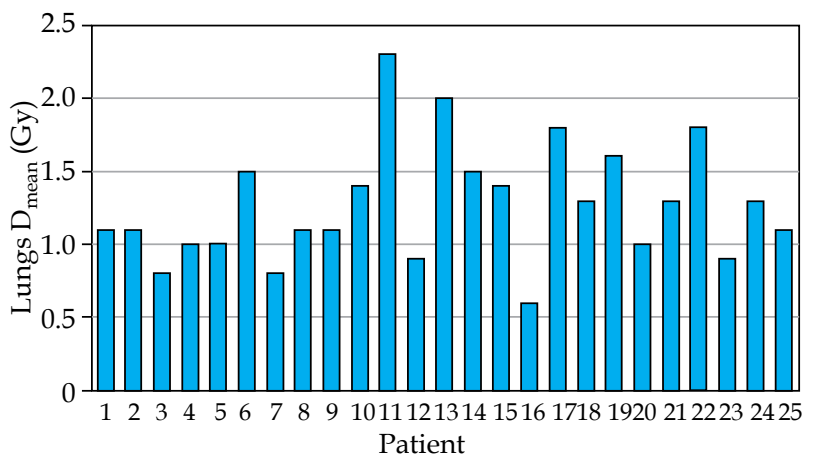

Fig. 5. $\mathrm{D}_{\text {mean }}$ values to the lungs for all of the 25 patients

$1.4 \mathrm{~Gy}\left(\mathrm{EQD}_{2}, 2.8 \mathrm{~Gy}\right)$. After 3 months of follow-up, no toxic effects of the skin were noted. Figure 3 shows the radiation dose exposed to the ribs. The maximum dose for this OAR was $50.1 \mathrm{~Gy}\left(\mathrm{EQD}_{2}, 80.3\right)$ at the acceptable maximum of $49.3 \mathrm{~Gy}\left(\mathrm{EQD}_{2}, 78.2\right)$. Therefore, the excess of the dose did not exceed $1 \mathrm{~Gy}\left(\mathrm{EQD}_{2}, 2 \mathrm{~Gy}\right)$. At five months after the treatment, patient number 16 presented no toxic effects.

Radiation exposure to the lung was estimated by two parameters: the maximum dose (20.4 Gy) (Figure 4) and the mean dose to the whole organ (2.7 Gy) (Figure 5). It was equivalent to $20.6 \mathrm{~Gy}$ and $1.8 \mathrm{~Gy}$ at $\mathrm{EQD}_{2}$ recalculation. In the present study, $\mathrm{D}_{\text {mean }}$ was lower than normal dose rate in all cases, while 6 patients presented overdosed $\mathrm{D}_{\max }$ by 0.7-7.4 Gy (0.4-5.5 Gy). After assessing of the overdosed volume, its location, and the mean dose, it was decided to make a course of brachytherapy. The median follow-up of these patients was 7 months (range, 4-11 months), during which no toxic effects were noted.

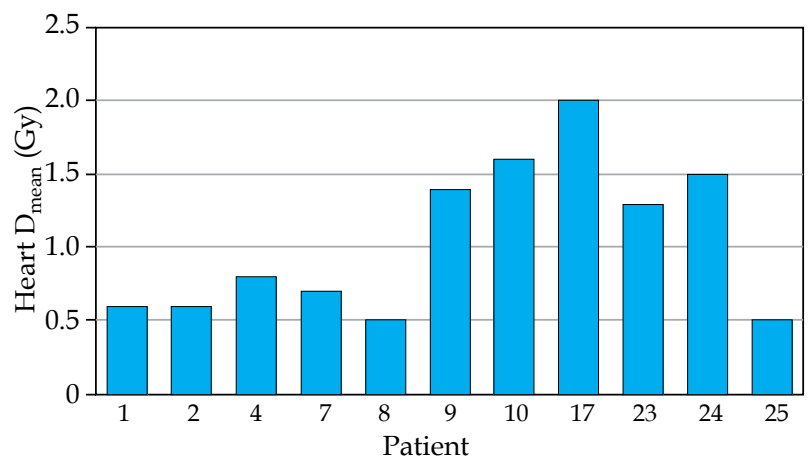

Fig. 7. $D_{\text {mean }}$ values to the heart for 11 patients

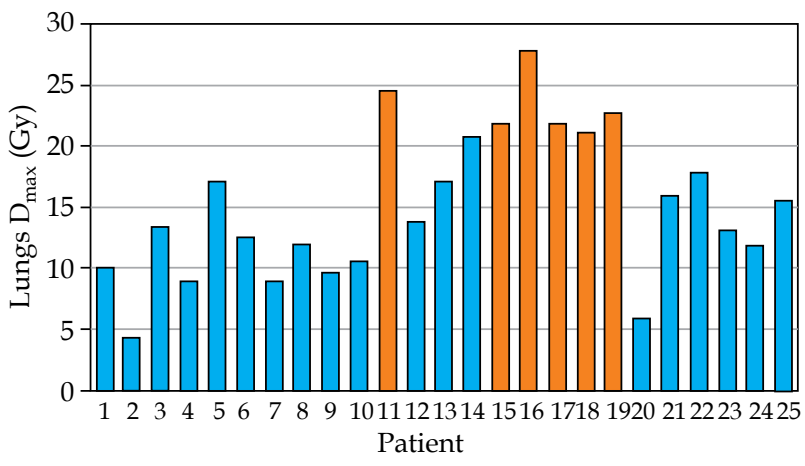

Fig. 4. $\mathrm{D}_{\max }$ values to the lungs for all of the 25 patients

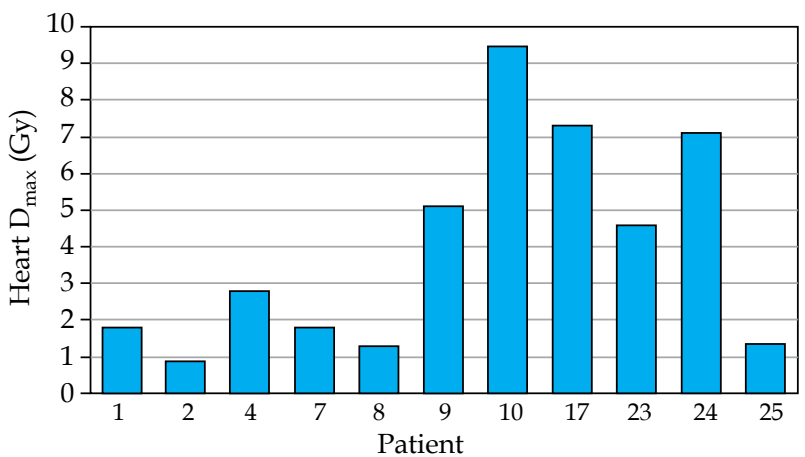

Fig. 6. $D_{\max }$ values to the heart for 11 patients

As described above, it was necessary to take into account the dose limits for the heart or liver depending on the tumor bed location. Firstly, the heart was contoured in the case of left breast, and sometimes when tumor was located in the inner quadrants of the right breast. The liver was taken as OAR, when the tumor bed was in the lower quadrants of the right breast. Also, the choice of these OARs was affected by the size of breast volume. However, the final decision was made on the CT study with introducers in place. There were heart $\mathrm{D}_{\max }$ and $\mathrm{D}_{\text {mean }}$ (Figures 6 and 7), and liver $D_{\max }$ (Figure 8) for those patients who required assessment of radiation exposure to this OARs. In all cases, the requirements of the protocol were followed.

\section{Discussion}

In our study, the dose-volume histograms contained information used to estimate the risk of radiation-induced toxic effect after APBI. The strongest link between

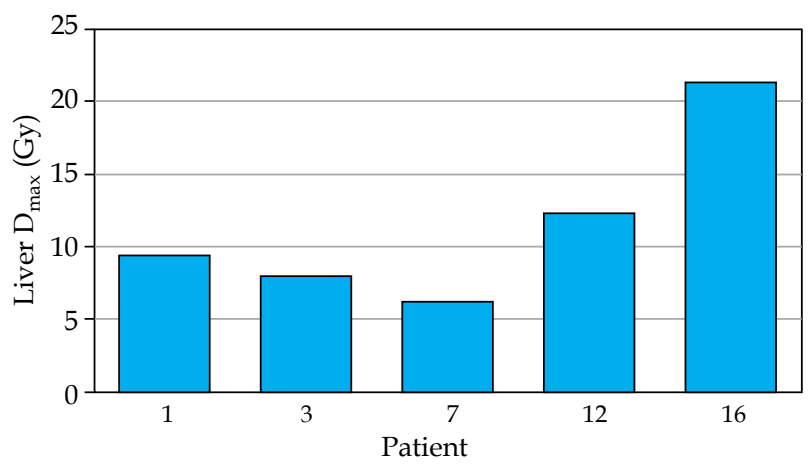

Fig. 8. $D_{\max }$ values to the liver for 5 patients 
the risk of radiotoxicity and DVH parameters was in the high-dose region. The risk could be quantitatively identified and prevented with DVH curves.

From an article of Roth et al. [18], it can be seen that with the use of the intraoperative mark, the mean value of $\mathrm{V}_{100}$ equals to $95.77 \%$. At the same time, the mean number of needles was 15 (without marks $\mathrm{V}_{100}=75.3 \%, \mathrm{~N}_{\text {needles }}=$ 11.3). In connection with the development of the method, at the initial stage, our $\mathrm{V}_{100}$ values were lower and affected the mean value. Further, we have refined the methods by determining the target and placement of introducers, which facilitated achieving almost $100 \%$ coverage of the $\mathrm{d}_{\text {ref: }}$. Compared to this study, we used fewer introducers (in our study, the mean value of $\mathrm{N}_{\text {needles }}$ was 4 ), which provided greater patient comfort during the therapy.

Ahmad et al. reported a mean value for $\mathrm{D}_{\max }$ to the skin of $30.9 \mathrm{~Gy}$, which was comparable with our results [19]. According to Sinnatamby et al., $\mathrm{D}_{\max }$ to the skin for all patients was $92.93 \%$, while the ribs accounted for only $20.05 \%$ of the prescribed dose [20]. The value of the skin dose is identical to ours, while the ribs' doses have a significant difference. Most likely is due to different methods for estimating the radiation exposure to this OAR. Oshaghi et al. compared the mean doses to the skin and ribs using a multicatheter brachytherapy and treatment with MammoSite [21]. With the multicatheter brachytherapy, they achieved 23 Gy and 22 Gy, respectively. At the same time, the mean dose to the skin was $25 \mathrm{~Gy}$ and to the ribs $35 \mathrm{~Gy}$ with MammoSite. These data show the advantages of multicatheter brachytherapy over single catheter approaches (including MammoSite). Lasota et al. published their data on the assessment of the absorbed doses to the skin drawn with different contouring methods [22]. One of them was to draw $2 \mathrm{~mm}$ inside and $2 \mathrm{~mm}$ outside the body contour (Skin$4 \mathrm{~mm}$ ). Another model was to expand $4 \mathrm{~mm}$ outside from the patient surface $\left(\mathrm{Skin}_{\mathrm{EXT}}\right)$. So, the dose for the first method was $81.02 \%$ of the prescribed dose, and $D_{\max }$ for $\mathrm{Skin}_{\mathrm{EXT}}$ was $62.59 \%$. However, in our opinion, it was more correct to take an indent of $2-5 \mathrm{~mm}$ (in our case, $3 \mathrm{~mm}$ ) inside from the body surface for the skin, obtaining a non-significant difference in values.

Yoshida et al. analyzed one case among 46 patients with rib fracture occurrence [23]. They used a fractionation model of $6 \times 6 \mathrm{~Gy}$. As a result, the toxic effect was identified when the total dose to the ribs was $54.6 \mathrm{~Gy}$. In our study, the maximum $\mathrm{D}_{\max }$ was $50.1 \mathrm{~Gy}$, but we should consider $\mathrm{EQD}_{2}$ value for these parameters. In the case of Yoshida et al., it was $132.1 \mathrm{~Gy}$, and in our study, EQD value was $80 \mathrm{~Gy}$. Thus, we acquired a significant difference with a recalculation. We compared our results with a study of Asai et al., where $\mathrm{D}_{\max }$ was $42.4 \mathrm{~Gy}$ per four fraction $\left(\mathrm{EQD}_{2}=115.3 \mathrm{~Gy}\right)$ [24]. In particular, comparisons were made between a whole breast radiotherapy (WBRT) and breast brachytherapy. For example, Smith et al. reported that rib fractures were $3.6 \%$ for WBRT and $4.5 \%$ for the brachytherapy [25]. The similar data were presented by Huo et al., with $1.3 \%$ for WBRT vs. $1.6 \%$ for brachytherapy. However, they noted that the risk of toxic effects with a multicatheter brachytherapy $(1.3 \%)$ was lower than with a single catheter $(1.8 \%)$ [26].

\section{Conclusions}

Irradiation of early stage breast cancer with APBI is a standard post-operative treatment worldwide. Today in Russia, it is only gaining momentum. Multicatheter brachytherapy has the advantage over other types because this is the only radiation therapy after conserving surgery lasting less than a week. There are some important points in the development of this method, including criteria for proper patient selection, catheter administration, dose optimization, and quality assurance of radiation therapy.

The study reports the first dosimetric outcomes of the breast cancer brachytherapy in our center. In general, they are suitable for the developed standards in our center and do not contradict international recommendations; each case should be considered individually.

Since the article is a review of the first results, with small number of patients, further studies on investigating toxic effects and survival are necessary.

\section{Disclosure}

Authors report no conflict of interest.

\section{References}

1. Bellanger $\mathrm{M}$, Zeinomar N, Tehranifar $\mathrm{P}$ et al. Are global breast cancer incidence and mortality patterns related to country-specific economic development and prevention strategies. J Glob Oncol 2018; 4: 1-16.

2. Fitzmaurice C, Allen C, Barber RM et al. Global, regional, and national cancer incidence, mortality, years of life lost, years lived with disability, and disability-adjusted life-years for 32 cancer groups, 1990 to 2015: A systematic analysis for the global burden of disease study. JAMA Oncol 2017; 3: 524-548.

3. International Agency for Research on Cancer (IARC) and World Health Organization (WHO). GLOBOCAN 2018: Breast. 2018. URL: http://gco.iarc.fr/today/data/ factsheets/cancers/20-Breast-fact-sheet.pdf (access date 15.11.2018).

4. Gancev Sh. Oncology: The manual for medical universities. OOO Medical information agency, Moscow 2006; 488.

5. Harat A, Harat M, Makarewicz R. Whole breast irradiation vs. APBI using multicatheter brachytherapy in early breast cancer - simulation of treatment costs based on phase 3 trial data. J Contemp Brachytherapy 2016; 8: 505-511.

6. Strnad V, Major T, Polgár C et al. ESTRO-ACROP guideline: Interstitial multicatheter breast brachytherapy as Accelerated Partial Breast Irradiation alone or as boost - GEC-ESTRO Breast Cancer Working Group practical recommendations. Radiother Oncol 2018; 128: 411-420.

7. Kaprin A, Starinsky V, Petrova V et al. The state of cancer care in Russia in 2016. MNIOI P.A. Herzen - branch of the National Medical Research Radiological Centre of the Ministry of Health of the Russian Federation, Moscow 2017; 236.

8. Mayadev J, Benedict S, Kamrava M. Handbook of image-guided brachytherapy. Springer International Publishing 2017; 622.

9. Latorre J, Galdós P, Buznego L et al. Accelerated partial breast irradiation in a single $18 \mathrm{~Gy}$ fraction with high-dose-rate brachytherapy: preliminary results. J Contemp Brachytherapy 2018; 10: 58-63.

10. Shah C, Vicini F, Shaitelman SF et al. The American Brachytherapy Society consensus statement for accelerated partial-breast irradiation. Brachytherapy 2018; 17: 154-170. 
11. Nose $\mathrm{T}$, Otani $\mathrm{Y}$, Asahi $\mathrm{S}$ et al. A Japanese prospective multi-institutional feasibility study on accelerated partial breast irradiation using interstitial brachytherapy: clinical results with a median follow-up of 26 months. Breast Cancer 2016; 23: 861-868.

12. Polgár C, Major T, Fodor J et al. High-dose-rate brachytherapy alone versus whole breast radiotherapy with or without tumor bed boost after breast-conserving surgery: seven-year results of a comparative study. Int J Radiat Oncol Biol Phys 2004; 60: 1173-1181.

13. Khan A, Vicini F, Brown S et al. Dosimetric feasibility and acute toxicity in a prospective trial of ultrashort-course accelerated partial breast irradiation (APBI) using a multi-lumen balloon brachytherapy device. Ann Surg Oncol 2013; 20: 1295-1301.

14. Ksuer-Dorner D, Berger D. The role of brachytherapy in the treatment of breast cancer. Breast Care 2018; 13: 157-161.

15. Owen JR, Ashton A, Bliss JM et al. Effect of radiotherapy fraction size on tumour control in patients with early-stage breast cancer after local tumour excision: long-term results of a randomised trial. Lancet Oncol 2006; 7: 467-471.

16. Strnad V, Hannoun-Levi JM, Guinot JL et al. Recommendations from GEC ESTRO Breast Cancer Working Group (I): Target definition and target delineation for accelerated or boost Partial Breast Irradiation using multicatheter interstitial brachytherapy after breast conserving closed cavity surgery. Radiother Oncol 2015; 115: 342-348.

17. Major T, Gutiérrez C, Guix B et al. Recommendations from GEC ESTRO Breast Cancer Working Group (II): Target definition and target delineation for accelerated or boost partial breast irradiation using multicatheter interstitial brachytherapy after breast conserving open cavity surgery. Radiother Oncol 2016; 116: 199-204.

18. Roth AM, Kauer-Dorner D, Resch A et al. Is oncoplastic surgery a contraindication for accelerated partial breast radiation using the interstitial multicatheter brachytherapy method? Brachytherapy 2014; 13: 394-399.

19. Ahmad S, Johnson D, Hiatt JR et al. Comparison of tumor and normal tissue dose for accelerated partial breast irradiation using an electronic brachytherapy eBx source and an Iridium-192 source. J Appl Clin Med Phys 2010; 11: 155-161.

20. Sinnatamby M, Nagarajan V, Sathyanarayana RK et al. Study of the dosimetric differences between ${ }^{192} \mathrm{Ir}$ and ${ }^{60} \mathrm{Co}$ sources of high dose rate brachytherapy for breast interstitial implant. Rep Pract Oncol Radiother 2016; 21: 453-459.

21. Oshaghi M, Sadeghi M, Mahdavi SR. A comparison of skin dose delivered with MammoSite and multicatheter breast brachytherapy. J Biomed Phys Eng 2013; 6: 200-207.

22. Lasota J, Kabacińska R, Makarewicz R. Dose estimation for different skin models in interstitial breast brachytherapy. J Contemp Brachytherapy 2014; 6: 200-207.

23. Yoshida K, Otani Y, Nose T et al. Case report of a dose-volume histogram analysis of rib fracture after accelerated partial breast irradiation: interim analysis of a Japanese prospective multi-institutional feasibility study. J Contemp Brachytherapy 2018; 10: 274-278.

24. Asai K, Shioyama Y, Nakamura K et al. Radiation-induced rib fractures after hypofractionated stereotactic body radiation therapy: risk factors and dose-volume relationship. Int J Radiat Oncol Biol Phys 2012; 84: 768-773.

25. Smith GL, Xu Y, Buchholz TA et al. Association between treatment with brachytherapy vs whole-breast irradiation and subsequent mastectomy, complications, and survival among older women with invasive breast cancer. JAMA Oncol 2012; 307: 1827-1837.

26. Huo J, Giordano SH, Smith BD et al. Contemporary toxicity profile of breast brachytherapy versus external beam radiation after lumpectomy for breast cancer. Int J Radiat Oncol Biol Phys 2016; 94: 709-718. 